\title{
Knowledge of cervical cancer screening among rural Indian women: a cross sectional study
}

\author{
SuryapriyaBalan Thovarayi ${ }^{1}$, Judith Angelitta Noronha ${ }^{2}$, Shobha. Nayak ${ }^{3}$ \\ ${ }^{1}$ MSc Nursing student, Manipal College of Nursing, Manipal University, Manipal, India \\ ${ }^{2} \operatorname{MSc}(N), M(P h i l), P h D$, Professor and HOD,Department of Obstetrics and Gynaecological Nursing, Manipal \\ College of Nursing, Manipal University, Manipal, India \\ ${ }^{3}$ MSc (N), Lecturer,Department of Obstetrics and Gynaecological Nursing, Manipal College of Nursing, \\ Manipal University, Manipal, India
}

\begin{abstract}
Cervical cancer is the fifth most common cause of cancer death in the world and the second largest cause of cancer mortality in India. Currently India accounts for $1 / 4^{\text {th }}$ of the global cervical cancer burden . Though the incidence of cervical cancer has declined in developed countries, in developing countries like India where women do not undergo routine cervical cancer screening it is still a major problem.

Purpose: This study was designed to assess the knowledge of cervical cancer, its symptoms, the Pap (Papanicolaou) smear test for screening and screening guidelines.

Methods: A cross sectional study was conducted among 407 women aged 21-65 years in a randomly selected village of udupitaluk in Karnataka. The data was collected using a valid knowledge questionnaire.

Findings: Majority of the study population $(98.5 \%, 401)$ had poor knowledge regarding cervical cancer screening. There was no apparent difference in the mean and standard deviation of knowledge scores in the

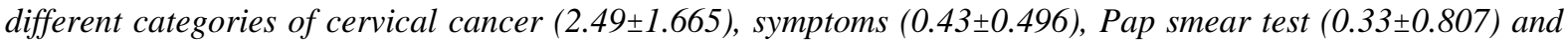
screening guidelines $(0.51 \pm 0.742)$. There is very poor knowledge of cervical cancer screening among women. Effective female education and mass screening are necessary for successful cervical cancer screening programme in India.
\end{abstract}

Keywords: knowledge, cervical cancer, screening, rural, Pap smear test, Indian women

\section{Introduction}

Cervical cancer is the number one cause of death in middle aged women and $20 \%$ of all cancer related deaths in women occur in India[1]. Cervical cancer is usually asymptomatic until nearby tissue is invaded by the cancerous cervical cells. The most common symptom is abnormal vaginal bleeding. However with adequate screening it can be detected in its early precancerous stage. Cervical cancer screening using the Papanicolaou (Pap) test prevents the development of cervical cancer by recognizing a precancerous state in the cervix and allows $90 \%$ of cervical cancers to be identified, treated and cured before it can spread.Pap smears have been shown to detect early cervical cellular abnormalities, thereby reducing morbidity and mortality from cervical cancer [2]. But successfully organized, population-based cervical cancer screening programmes have not yet been implemented in most developing countries, despite the greatest burden of cervical cancer in these countries which is largely related to poverty, lack of resources and infrastructure and disenfranchisement of women [3]. Since early detection predicts better prognosis, one of the most effective ways of preventing and controlling cervical cancer is regular screening and early diagnosis.

India has a population of 366.58 million women aged 15 years and older who are at risk of developing cervical cancer. As reported by Tamil Nadu Systems Project with a Crude Incidence Rate of 23.5, current estimates indicate that every year 134,420 Indian women are diagnosed with cervical cancer with the figure for new cervical cases projected to reach 203,757 by 2025 [4]. It is estimated that at present approximately 72,825 women die due to cancer of the cervix. By 2025, this number is projected to rise to 115,171. A lack of effective screening programs aimed at detecting and treating precancerous conditions is a key reason for the much higher cervical cancer incidence in developing countries. A study in a developing country showed that $70.6 \%$ of the respondents lacked knowledge of cancer of cervix and only $15.5 \%$ of the respondents had knowledge of cervical cancer screening [5]. Donta et al. (2012) reported that awareness of cervical cancer and Pap smear test among couples is low[3]. Dhamija et al. (1993) reported that younger women have better awareness and knowledge about cervical cancer and related information. Literacy status and exposure to family planning are influential in creating awareness about cancer cervix[6]

Cervical cancer causes loss of productive life both due to early death as well as prolonged disability ${ }^{[7]}$ In India, the Years of Life Lost (YLL) due to cervical cancer were 936.3 in 2000, being among the highest in the world, greater than the YYLs caused by any other cancer in India, and constituting almost $4 \%$ of total YYLs due 
to all causes in India [8]. Among women aged 25-64 years, who tend, in India, to be the sole caretakers of the house \& family, and in some cases significant contributors to the family income, this mortality burden poses a heavy economic burden on families as well the country. Additionally, the high medical costs that are incurred by families due to cervical cancer (especially since most cases in developing countries are diagnosed at advanced stages when treatment is costly but prognosis poor), further impoverish individuals and communities [9].

The aim of this study was to explore knowledge of rural women in three village of Udupi, India regarding cervical cancer and cervical cancer screening. Additionally, we attempted to identify association between the knowledge level and some sociodemographic parameters.

\section{Materials and Methods}

A cross sectional survey design was used for this study. This study was conducted in rural areas of Udupitaluk, India. Udupitaluk has a rural population of 158651 females.There are 22 primary health centre (PHCs) in Udupitaluk. Out of these, the PHC Moodebettu was selected conveniently. The reason for selecting this particular PHC was that the population characteristics were similar and economy of time and ease of access. Under this PHC there are 5 villages. 3 villages were selected randomly from these villages namely Moodebettu, Kote and Yenegudde. A structured questionnaire was used to collect the data. The questionnaire was pretested using 7 women and was examined by experts for content validity. The questionnaire was then administered to 20 different women. When the data were subjected to Kudor Richardson's formula, the reliability was found to be significant at 0.05 levels. The questionnaire was interviewer administered to married women in the age group of 21-65 years residing in the above mentioned villages during January to February 2013. 157 women from Moodebettu, 150 from Yenegudde and 100 from Kote were selected through house to house survey. Women who had undergone total hysterectomy were excluded. A written informed consent was obtained from each of the participants. Anonymity of the subjects and confidentiality of the data were maintained. A total of 407 women were included in the study. The first part of the questionnaire collected sociodemographic information in 3 sections which were demographic, biological and source of health care related information. The remaining part contained questions on most prevalent cancer type among the Indian women, usual age of occurrence of cervical cancer, risk factors for it, symptoms, role of sexual activity in causing the cancer, Pap Smear Test, HPV and screening guidelines.

The data collected were analysed using SPSS version 11 statistical programme. Descriptive statistics and $\chi^{2}$-test for difference in proportions were used for univariate analysis. A $p$-value of 0.05 or less was considered statistically significant.

\section{Results}

The data presented in Table 1 shows that the mean age of the women in the study population was 43.17 \pm 13.43 with the age range from $21-65$ years. A majority $(151,37.1 \%)$ have high school education. Majority $(207,40 \%)$ have monthly income less than 5000 rupees. Most of the subjects $(315,77.3 \%)$ were married within $18-25$ years. Majority of the women belong to joint family $(218,53.6 \%)$

Data presented in Table 2 shows that women who had had 2 pregnancies comprised $42.8 \%$ (174) of the study population. Majority of the subjects (45\%) had 2 children with most of them $(305,76.1 \%)$ having the first child between the ages of 18-25 years. Majority of the subjects $(337,82.8 \%)$ had no history of abortions/MTPs. Majority of them $(406,99.8 \%)$ had only a single sexual partner and majority $(227,55.7 \%)$ had their first sexual experience within 13-20 years. Only $2(0.5 \%)$ had a history of smoking or tobacco chewing. There was no family or personal history of cervical cancer or STDs. $2(0.5 \%)$ of the women reported a history of sexual abuse. The findings shows that the source of health related information for $91.4 \%$ (372) of the subjects was family members, $5.2 \%$ (21) from media and only $2.5 \%$ (10) from health personnel.

Majority of the study population $(98.5 \%, 401)$ had poor knowledge regarding cervical cancer screening. Only $1.5 \%$ (6) had good knowledge.

The findings reveals that 173 women had good knowledge about cervical cancer; 177 had good knowledge about the symptoms of cervical cancer and 161 had good knowledge about screening guidelines; but only 88 had good knowledge about the Pap smear test.(Table 4)

Data in Table 5 showed that there was significant association between knowledge and selected variables like education, income, age at first sexual intercourse and source of health related information. It was found that the subjects with good knowledge about cervical cancer were better educated. Subjects who were married after the age of 18 years and had their first sexual experience after the age of 21 years had good knowledge. Women who lived in nuclear families had good knowledge.

\section{Discussion}

This study has shown that women in our region lack knowledge of cervical cancer and its prevention. The findings of the present study show that $98.5 \%$ had poor knowledge regarding cervical cancer screening. 
Similar findings were found in a study done in Kerala among 809 women where only half of the study population was aware of symptoms and the majority (89.2\%) were unaware of the risk factors for cervical cancer [10] and Nigeria (29.4\%) [5]. This is also supported by another study done by in Texas done to assess the knowledge regarding Pap test in which women of low socioeconomic status had poor understanding of Pap testing [11].

It is a known fact that the knowledge of cervical cancer prevention is very poor in developing nations because of poor education and deficient awareness programmes. This is reflected in this study with higher proportion of the more educated showing better knowledge compared with the less educated. Though $43 \%$ had good knowledge about symptoms and 39\% had good knowledge about screening guidelines only $21 \%$ knew about the Pap smear test. Similar findings were found in a study done in Kolkata where there was good knowledge about cervical cancer but poor knowledge about the Pap test [12]. Most of the women in this study (91\%) got their health related information from their family members which could be one of the reasons for the poor knowledge regarding cervical cancer.

A limitation of the study may be the potential for information bias which exists in the accurate disclosure of personal information. Women may be hesitant to report having multiple sexual partners, their age at initiation of sexual activity, and history of STDs. We attempted to minimize this bias by ensuring participant confidentiality. Also this study was conducted in villages of a primary health centre that was conveniently selected due to financial and time constraints.

In conclusion, undergoing regular cervical cancer screening is important in the prevention of invasive cervical carcinoma. The knowledge about cervical cancer screening is very poor among rural Indian women. There is need for organized education programmes to create awareness about cervical cancer and cervical cancer screening among Indian women.

Declaration of interest: The authors report no conflicts of interest. The authors alone are responsible for the content and writing of the paper.

\section{References}

[1]. Eddy DM. Screening for cervical cancer. Annals of Internal Medicine .1990,113:214-216.

[2]. Bhatla N. Ending cervical cancer in Asia: regional burden of disease. Cervical Cancer Action "Champions" Webinar. 2012.

[3]. Donta B, Begum S, Saritha N, Naik DD, Mali BN, BandiwadekarA.Awareness of Cervical Cancer among Couples in a Slum Area of Mumbai. Asian Pacific Journal of Cancer Prevention 2012,13: 4901-4903.

[4]. Tamil Nadu Systems Projec.. Department of Health and Family Welfare. Screening for cervical and Breast Cancer. Government of Tamil Nadu. 2005 (Available from http://www.tnhsp.org/screening-cervical-cancer-and-breast-cancer).

[5]. Nwankwo K.C, Aniebue U, Aguwa E.N, Anarado AN, Agunwah E. Knowledge attitudes and practices of cervical cancer screening among urban and rural Nigerian women: a call for education and mass screening. European Journal of Cancer Care.2011, 20:362367.

[6]. Dhamija S, Sehgal A, Luthra UK, SehgalK.Factors associated with awareness of cervical cancer in a community. Perspectives in Public Health.1998, 113: pp184-186.

[7]. WHO/ICO. Information Centre on Human Papilloma Virus (HPV) and Cervical Cancer Human Papillomavirus and Related Cancers in India. Summary Report.2009 (Available at http://www.who.int/hpvcentre/en/)

[8]. Yang BH, Bray FI, Parkin M, Sellors JW, Zhang ZF. Cervical cancer as a priority for prevention in different world regions: An evaluation using years of life lost. International Journal of Cancer.2004, 109:418-424.

[9]. Satija A. Cervical cancer in India. WHO Comprehensive Cervical Cancer Control.2006 (Available from sancd.org/uploads/pdf/cervicalcancer).

[10]. Aswathy S, Quereshi MA, Kurian B, Leelamoni K. Cervical cancer screening: Current knowledge and practice among women in a rural population of Kerala, India. Indian Journal of Medical Research 2012. 136: pp 205-210.

[11]. Breitkopf CR, Pearson HC, Breitkopf DM. Poor knowledge regarding the Pap test among low-income women undergoing routine screening. Perspectives on Sexual and Reproductive Health 2005.37: pp78-84.

[12]. Saha A, Chaudhury AN, Bhowmik P, Chatterjee R. Awareness of cervical cancer among female students of premier colleges in Kolkata, India.Asian Pacific Journal of Cancer Prevention.2010, 11:1085-1090 
Table 1. Socio-demographic characteristics of 407 women surveyed

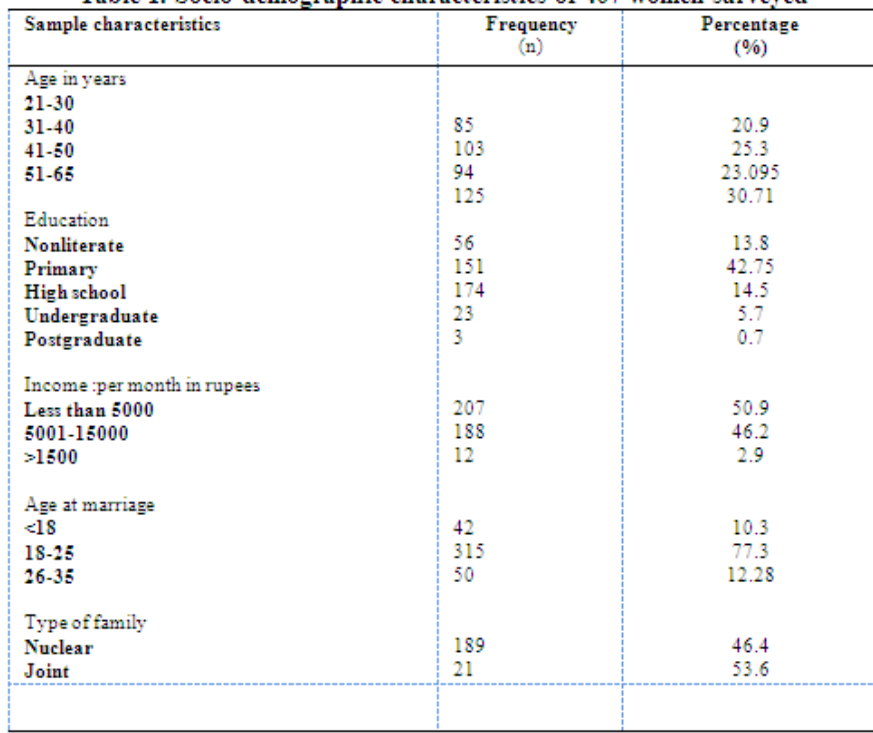

Table 2: Frequency and percentage distribution of biological variables $\mathrm{N}=\mathbf{4 0 7}$

\begin{tabular}{|c|c|c|}
\hline Sample characteristics & Frequency & Percentage \\
\hline \multirow{2}{*}{\multicolumn{3}{|c|}{$\begin{array}{l}\text { I. BIOLOGICAL } \\
\text { Number of pregnancies }\end{array}$}} \\
\hline & & \\
\hline None & 6 & 1.5 \\
\hline 1 & 69 & 17 \\
\hline 2 & 174 & 42.8 \\
\hline 3 & 109 & 26.8 \\
\hline$>3$ & 49 & 26.8 \\
\hline \multicolumn{3}{|l|}{ Age during first child birth } \\
\hline$<18$ & 20 & 5.0 \\
\hline $18-25$ & 305 & 76.1 \\
\hline $26-35$ & 75 & 18.7 \\
\hline$>35$ & 1 & 0.2 \\
\hline \multicolumn{3}{|l|}{ Number of living children } \\
\hline None & 7 & 1.7 \\
\hline 1 & 84 & 20.6 \\
\hline 2 & 178 & 43.7 \\
\hline 3 & 108 & 26.5 \\
\hline$>3$ & 30 & 7.4 \\
\hline \multicolumn{3}{|l|}{ Number of MTPs/abortions } \\
\hline None & 337 & 82.8 \\
\hline 1 & 43 & 10.6 \\
\hline$\geq 2$ & 27 & 6.6 \\
\hline \multicolumn{3}{|c|}{ Age at first sexual experience } \\
\hline $13-20$ & 227 & 55.7 \\
\hline $21-32$ & 180 & 44.2 \\
\hline \multicolumn{3}{|l|}{ Number of sexual partners } \\
\hline 1 & 406 & 99.8 \\
\hline 2 & 1 & 0.2 \\
\hline \multicolumn{3}{|c|}{ History of smoking/tobacco chewing } \\
\hline No & 405 & 99.5 \\
\hline Yes & 2 & 0.5 \\
\hline \multicolumn{3}{|l|}{ Experienced sexual abuse } \\
\hline Yes & 2 & 0.5 \\
\hline No & 405 & 99.5 \\
\hline
\end{tabular}


Table 3: Frequency and percentage distribution of knowledge score on cervical cancer screening $\mathbf{N}=\mathbf{4 0 7}$

\begin{tabular}{clll}
\hline Knowledge & Range of Score & Frequency & Percentage \\
\hline Poor & $1-10$ & 401 & 98.5 \\
Good & $11-20$ & 6 & 1.5 \\
\hline
\end{tabular}

Table4: Frequency and percentage distribution of knowledge score in different categories

\begin{tabular}{llccccc} 
& & & & \multicolumn{2}{c}{$\mathbf{n = 4 0 7}$} \\
\hline Knowledge & Poor & Good & Range & Mean & Median & Standard Deviation \\
\hline Cervical Cancer & 234 & 173 & 10 & 2.49 & 2 & 1.665 \\
Symptoms & 230 & 177 & 1 & 0.43 & 0 & 0.496 \\
Pap smear test & 318 & 88 & 5 & 0.33 & 0 & 0.807 \\
Screening Guidelines & 246 & 161 & 4 & 0.51 & 0 & 0.742 \\
\hline
\end{tabular}

Table 5: Association between knowledge and selected variables

\begin{tabular}{|c|c|c|c|c|c|}
\hline Variable & Poor knowledge & $\begin{array}{c}\text { Good } \\
\text { knowledge }\end{array}$ & $\mathbf{X}^{2}$ & df & p-value \\
\hline \multicolumn{6}{|l|}{ Age in years } \\
\hline $21-30$ & 84 & 1 & & & \\
\hline $31-40$ & 101 & 2 & & & \\
\hline $41-50$ & 92 & 2 & $0.909^{*}$ & 3 & 0.823 \\
\hline $51-65$ & 124 & 1 & & & \\
\hline \multicolumn{6}{|l|}{ Education } \\
\hline None & 56 & 0 & & & \\
\hline Primary & 150 & 1 & & & \\
\hline High school & 115 & 0 & & & \\
\hline Higher secondary & 59 & 0 & $25.415^{*}$ & 5 & $0.000 * *$ \\
\hline Undergraduate & 19 & 4 & & & \\
\hline Postgraduate & 2 & 1 & & & \\
\hline \multicolumn{6}{|l|}{ Income } \\
\hline$<5000$ & 205 & 2 & & & \\
\hline $5001-15000$ & 186 & 2 & $7.012^{*}$ & 2 & $0.030 * *$ \\
\hline$>15000$ & 10 & 2 & & & \\
\hline \multicolumn{6}{|l|}{ Age at marriage } \\
\hline$<18$ & 42 & 0 & $5.924^{*}$ & 2 & $0.05^{* *}$ \\
\hline $18-25$ & 312 & 3 & & & \\
\hline $26-35$ & 47 & 3 & & & \\
\hline \multicolumn{6}{|l|}{ Age at last child birth } \\
\hline $18-25$ & 196 & 2 & $0.184^{*}$ & 1 & 0.668 \\
\hline $26-35$ & 199 & 3 & & & \\
\hline \multicolumn{6}{|c|}{ Age at first sexualexperience } \\
\hline \multicolumn{6}{|l|}{$13-20$} \\
\hline $21-32$ & 180 & 0 & $7.077^{*}$ & 1 & $0.008 * *$ \\
\hline Type of family & 221 & 6 & & & \\
\hline \multicolumn{6}{|l|}{ Nuclear } \\
\hline \multirow[t]{2}{*}{ Joint } & 184 & 5 & & & \\
\hline & 217 & 1 & $3.562 *$ & 1 & 0.059 \\
\hline \multicolumn{6}{|l|}{ Experienced sexual abuse } \\
\hline No & 2 & 0 & & & \\
\hline $\begin{array}{l}\text { Source of health related } \\
\text { information }\end{array}$ & 399 & 6 & $0.60^{*}$ & 1 & 0.807 \\
\hline \\
\hline Health professionals & 370 & 2 & & & \\
\hline Media & 8 & 2 & & & \\
\hline Other & 21 & 0 & $22.070 *$ & 3 & $0.000 * *$ \\
\hline & 2 & 2 & & & \\
\hline
\end{tabular}

*likelihood ratio was used $* * \mathbf{P}<0.05$ 